\title{
Dissociation of cortical steady potential shifts from mass action potentials in cats awaiting food rewards
}

\author{
PATRICK J. SHEAFOR and VERNON ROWLAND †† \\ Department of Psychiatry, School of Medicine, \\ Case Western Reserve University, Cleveland, Ohio 44106
}

\begin{abstract}
Cortical steady potential shift (SPS) and mass unit activity, derived from the same transcortical hybrid electrodes, were recorded in cats undergoing single alternation (SA) training. A 10-sec tone CS, occurring every $2 \mathrm{~min}$, was alternately nonreinforced $(\mathrm{N})$ and reinforced $(\mathrm{R})$ with an intraoral food US. Results revealed: (1) Acquisition of a recurrent SPS pattern consisting of a gradual 200-300 microV shift during the pre- $R$ ( $N$ to $R$ ) interval followed by post- $R$ shifting of opposite polarity during the $R$-to- $N$ interval. (2) The pre-R SPS became dissociated from the integrated multiple unit (IMU) measure, but the post-R SPS remained associated with the IMU. (3) The CS elicited a conditional IMU response (cortical CR) when it signaled the US ( $R$ trial) but not when it signaled no US ( $N$ trial). The SPS pattern may reflect context-conditioned "expectancy" states (not detectable in IMU recording) postulated to "gate" the IMU CR on and off alternately.
\end{abstract}

Steady potential shifts (SPS) are of interest as possible endogenous electrical integrations of multicellular activity (e.g., Rebert, 1969, 1973a, b; Rowland \& Anderson, 1971; Rowland \& Dines, 1973) and as electrophysiologic correlates of learning (e.g., Chiorini, 1969; Donchin, Otto, Gerbrandt, \& Pribram, 1971; Irwin \& Rebert, 1970; Marczynski, 1972; Rebert, 1972, 1973b; Rebert \& Irwin, 1969; Rowland, 1967, 1968; Rowland \& Anderson, 1971; Steinmetz \& Rebert, 1972).

In order to study simultaneously the three major extracellular patterns of electrical activity, Rowland and Dines (1973) developed a recording system which provides the SPS, the oscillatory activity (ECoG), and an electronic integration of multiple unit (IMU) activity, all derived from the same hybrid electrodes. Their observations and those of Rebert $(1969,1973 a)$ have demonstrated consistent correlations of the IMU measure with short duration $(1-5 \mathrm{sec})$ SPS phenomena, and with transient ECoG and lateral geniculate events, e.g., orienting responses, saccade-related lambda waves, evoked potentials, and synchronous high-voltage slow activity. However, recent observations from this laboratory (Rowland, 1974) suggests that cats trained to await regularly scheduled food rewards may acquire anticipatory graded cortical SPSs up to minutes

*This research was supported by the Grant Foundation and by an NIMH Career Grant (MH-12035-05) to Vernon Rowland. Portions of the data were reported in a paper read at the Third Annual Meeting of the Society for Neuroscience, San Diego, California, November 1973.

tThis research was supervised by the second author and carried out by the first author during his appointment as a Postdoctoral Fellow in the Department of Psychiatry, Case Western R eserve University School of Medicine.

+ T equests for reprints should be addressed to Vernon Rowland, Department of Psychiatry, Case Western Reserve University School of Medicine, Cleveland, Ohio 44106. in duration which are not accompanied by corresponding change in IMU level. In addition, since Kuffler's initial invertebrate studies (Kuffler \& Nicholls, 1966), a number of investigations (e.g., Karahashi \& Goldring, 1966; Grossman et al, 1969; Somjen, 1970; Ransom \& Goldring, 1973a, b, c) have confirmed the contribution of presumed glial cells to the extracellularly recorded cortical and spinal cord SPS. Although these studies present an alternative to considering the origin of SPS as exclusively neuronal, nevertheless, the SPS dynamics are seen to be completely dependent on rates of neuronal activity as reflected in the extracellular potassium levels.

The purpose of this research was to extend Rowland's initial observations by assessing the relationships between cortical SPS and IMU in cats learning a single-alternation schedule in a Pavlovian reward conditioning situation. A well-documented behavioral finding is that single alternation (SA) of nonreinforced $(\mathrm{N})$ and reinforced $(\mathrm{R})$ presentations of the same CS (i.e., NRNR ...) leads eventually to lower levels of responding to the CS on $\mathrm{N}$ than on $\mathrm{R}$ trials (e.g., Poulos, Sheafor, \& Gormezano, 1972). In order to explain different responding to the same CS, behavioral theorists postulate different "stimulus aftereffects" of $\mathrm{N}$ and $\mathrm{R}$ trials (Capaldi, 1967). According to some theorists (cf. Amsel, 1967; Bolles, 1972; Rescorla \& Wagner, 1972), expectancies of nonreinforcement and reinforcement become differentially conditioned to $\mathrm{R}$ vs $\mathrm{N}$ trial aftereffects, respectively, and serve alternately to inhibit and facilitate responding to the same CS. Translating these concepts into Pavlovian (1927) terminology, one would expect to observe the acquisition of recurrent alternating cycles of cortical inhibition and excitation relating to the contexts differentiating $\mathrm{N}$ and $\mathrm{R}$ 
presentations of the CS. Accordingly, the questions posed were whether cats learning an SA schedule would acquire recurrent alternating cycles of cortical inhibition and excitation, whether these patterns would be reflected in the SPS and, if so, what relationships would obtain between SPS and IMU measures.

\section{METHOD}

\section{Subjects and Restraint}

Ss were six adult cats obtained from the University animal facility. After 2 weeks adaptation to new home cages, and prior to surgery, the animals were trained with food reinforcement to remain seated quietly in a stock restraint.

\section{Surgery \\ Under sodium pentobarbital anesthesia, either single electrodes or arrays having two to five recording points were implanted transcortically from pial surface (S) to depth (D) aimed at the 6th layer in posterior marginal, anterior ectosylvian, and pre- and postcruciate gyri. Reference and ground electrodes were placed either in or on skull bone along the midline from anterior (over frontal sinus) to posterior (occipital area). Stigmatic, reference, and ground electrodes were of identical hybrid construction. An electrically insulated 13-ga cannula was implanted transnasally through the hard palate to provide for intraoral delivery of food reward. Resistance changes across its intraoral terminus with licking provided a lickometer record.}

\section{Electrodes, Amplifiers, and Filters}

Electrodes were of a hybrid construction with electrical characteristics extensively detailed elsewhere (Rowland \& Dines, 1973). In brief, the electrodes consist of a platinized Pt-Ir tip welded to an $\mathrm{Ag}-\mathrm{AgCl}$ coil carried inside a capillary pipette. The beveled capillary tip is $250-400$ microns in diam. The platinized segment extends 200 microns beyond the capillary tip, tapering from 50 microns diam at the point of emergence down to 10 microns at the end. A saline-agar conductive bridge, air-free, surrounds the Pt-Ir, $\mathrm{Ag}-\mathrm{AgCl}$ sections contained within the capillary and is sealed off at the upper end. This electrode provides an impedance of $10,000 \mathrm{ohms}$ for frequencies below $10 \mathrm{~Hz}$; and of $1,000 \mathrm{ohms}$ or less for frequencies of $800 \mathrm{~Hz}$ and higher. Electrode drift is $1 \mathrm{microV} / \mathrm{min}$ or less and free of fluctuation from the platinum segment.

Signals from these electrodes are conducted to differential input amplifiers (Philbrick B25C), temperature matched, with input dc resistance of $10^{12} \mathrm{ohms}$ and impedance at $10 \mathrm{kHz}$ of $10^{7} \mathrm{ohms}$. The combined signal is further amplified and filtered in two basic frequency ranges for display. One, for the steady potential shifts and oscillatory electrocorticogram is from dc to $50 \mathrm{~Hz}$ with the upper-frequency range usually attenuated by further filtering at the ink writer to half-amplitude frequency response at $1.5 \mathrm{~Hz}$. The other frequency range is down $6 \mathrm{~dB}$ at $800 \mathrm{~Hz}$ and $2.6 \mathrm{kHz}$. Contamination of the mass unit data by slower activity (ECoG) was precluded by the low end of this range being down $25 \mathrm{~dB}$ at $500 \mathrm{~Hz}$. Peak-to-peak amplitudes of system noise in this channel averaged 5 microV with shorted input. The average background mass unit activity was 10 microV, and increments in the amplitudes of the raw mass unit activity with activation of the brain ranged up to 30 microV. The mass unit activity is passed through an integrator (capable of both frequency and amplitude response) for display as an integrated mass unit (IMU) record. Integrator decay time constant is $40 \mathrm{msec}$. The ink-written display of this integration is usually smoothed for purposes of slow chart recording by filtering the power amplifiers down to half-amplitude frequency response at $1.5 \mathrm{~Hz}$.

\section{Training}

For five of the six Ss, training was begun within 1 to 2 weeks following surgery and was carried out with the cat restrained and placed in a lighted, sound-resistant, modified refrigerator shell with a continuous white-noise background of approximately $75 \mathrm{~dB}$ SPL (re 0.0002 dynes $/ \mathrm{cm}^{2}$ ). Under the single alternation (SA) schedule, a 10-sec tone CS $(1,000 \mathrm{~Hz}, 10 \mathrm{~dB}$ above the white-noise background) was presented every $2 \mathrm{~min}$; every other CS was paired with an 8-cc intraoral feeding during the last $5 \mathrm{sec}$ of the CS. The food US was fishmeal (fish flavor Puss'n Boots) homogenized in evaporated milk and was delivered intraorally by a peristaltic pump. Ss, maintained at $85 \%-95 \%$ of ad lib weight, received one training session per day. Each session began with a nonreinforced tone $(\mathrm{N})$ followed 2 min later by a reinforced tone $(\mathrm{R})$, and the $\mathrm{N}-\mathrm{R}$ sequence was repeated from 20 to 50 times per session. The sixth S (172) differed from the others in having had a previous experimental history and being trained to a cylindrical restraint with regularly scheduled food reinforcements delivered to a small cup $1 / 2$ in. in front of its nose; also, its recording reference was in a lateral ventricle. This $S$, trained to classical temporal conditioning, was not on the SA schedule and was exposed to longer interreinforcement intervals than those used in the SA training. Selected Ss were tested for effects of varying the intervals of the training schedule and of omission of either CS or US over a series of trials following acquisition.

\section{RESULTS}

Acquisition of SPS pattern was obtained from 4 of 6 Ss, involving 8 of the 24 cortical electrode arrays implanted. The 16 arrays that failed lost signal largely owing to implant deterioration, either from cortical trauma or electrode failure. Arrays in which multiple electrodes penetrated the cortex at one locus tended to deteriorate rapidly, presumably from interference with pial circulation.

Four of the eight informative arrays were bipolar surface (S) to depth (D) derivations (1.5 mm tip separation) bilaterally placed in anterior ectosylvian (AE) and posterior marginal (PM) gyri of S 62. Figure 1 illustrates the acquisition of a graded pattern of SPS over the first 20 days of training under the SA schedule of this S. The SPS pattern obtained by Day 19 was maximally positive at, or just prior to, the occurrence of the $\mathrm{N}$ tone, represented at the left of the figure. Following the $\mathrm{N}$ tone, a graded increasingly negative SPS of 200-300 microV was observed during the 2-min period leading up to the $\mathrm{R}$ tone. Maximum negativity was reached during or closely following the $\mathrm{R}$ tone, and a graded positive SPS of equal amplitude ensued in the next 2-min period. The general features of the acquired pattern of SPS in the four successful Ss may be characterized in terms of four phases in relation to reinforcement: (1) prereinforcement (pre-R) negativity; (2) during reinforcement, a slight further negativity; (3) postreinforcement (post-R) positivity; and (4) in the case of longer intervals, a holding or transition phase between the termination of the post-R positive and onset of the pre- $R$ negative phases.

The pre- $R$ negativity, post-R positivity SPS pattern 


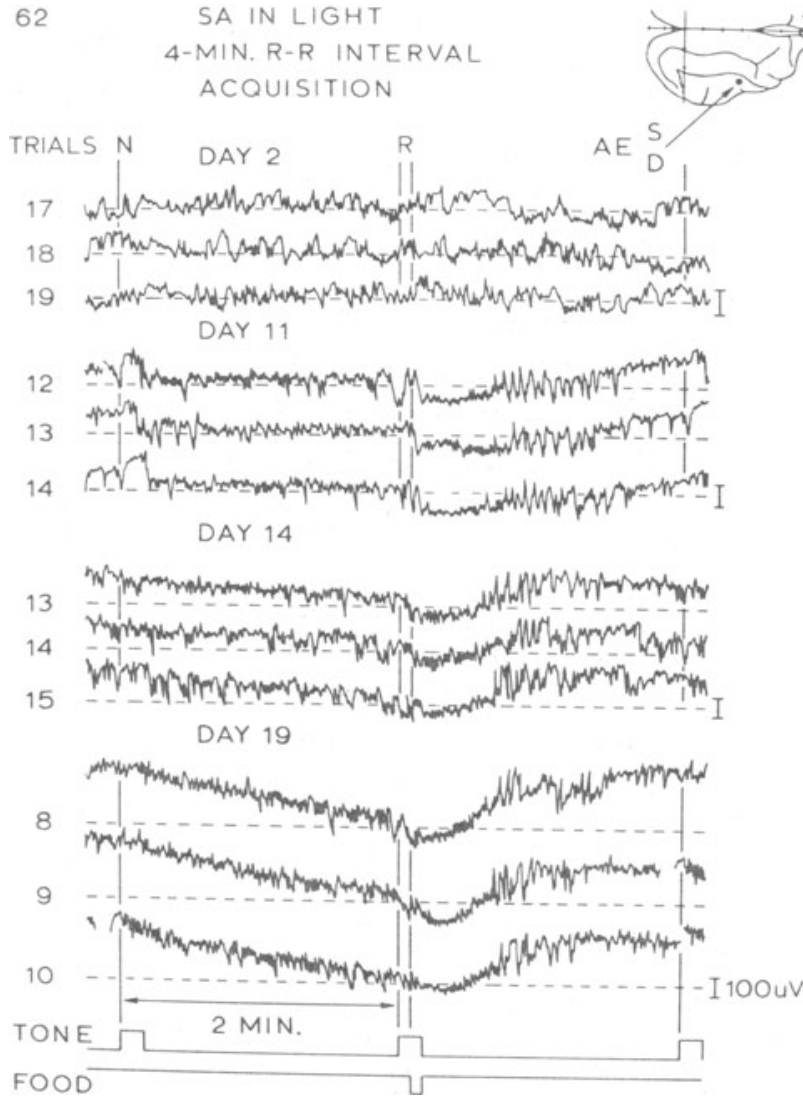

Fig. 1. Representative ECoG tracings of three consecutive trials taken from each of the days indicated illustrate acquisition of the SPS pattern derived from anterior ectosylvian gyrus (AE) in $S 62$ during $S A$ training. $S=$ cortical surface; $D=$ cortical depth $1.5 \mathrm{~mm}$ below S. All tracings derived S-D. ECoG passband is from dc to half-amplitude frequency response at $1.5 \mathrm{~Hz}$ in all figures except Fig. 8. Negativity is downward in this and all other figures.

characteristic of late acquisition contrasts sharply with the earlier performance observed on Day 2, when cortical response was not discerned, even to food. The tracings from Days 11 and 14 illustrate the gradual development of the later SPS pattern. On Day 11, with response to food well established, a graded post-R positive SPS of approximately $150 \mathrm{microV}$ which reached maximum positivity during the $\mathrm{N}$ tone was abruptly terminated by a 50-100 microV negative SPS at, or around, the point of $\mathrm{N}$-tone offset. During the following pre-R interval, the SP held flat until the next $\mathrm{R}$ tone. Day 14 tracings differ significantly from those of Day 11 in the transformation of the abrupt negative SPS at N-tone offset into a graded negative SPS distributed over the entire 2-min pre- $R$ interval. The main difference between Day 14 and Day 19 tracings is not in a changed pattern of shift, but in increased slope of the SPS.

Figure 2 exemplifies the relationships of the SPS patterns of Fig. 1 to the integrated multiple units (IMU) measure during each acquisition stage. The Day-2 pair of ECoG-IMU tracings reveals a correlation of the more sustained features of the two measures. An increase in IMU was accompanied by a negative SPS, a decrease by a reversal of SPS. This kind of SPS-IMU relationship is termed SPS-1. (It is readily observed by visual inspection and no attempt was made to evaluate covariation of the less sustained or very transient events in the ECoG and IMU, i.e., of less than $0.5 \mathrm{sec}$ duration, except for conspicuous events such as the lambda wave or synchrony bursts.) With continued acquisition training, a gradual dissociation of the two measures was observed as the pre-R-negative/post-R-positive SPS pattern developed. Specifically, by Day 11 the abrupt and sustained 50-100 microV negative SPS following N-tone offset was accompanied by only a brief increase in IMU level. This is a significant departure from the SPS-1 relationship in which such a sustained change in SP level would be accompanied by a correspondingly sustained increase in IMU level.

Day 14 records show a dissociation of the ECoG-IMU measures of the kind termed SPS-2. The acquired monotonic and smoothly graded negative SPS of

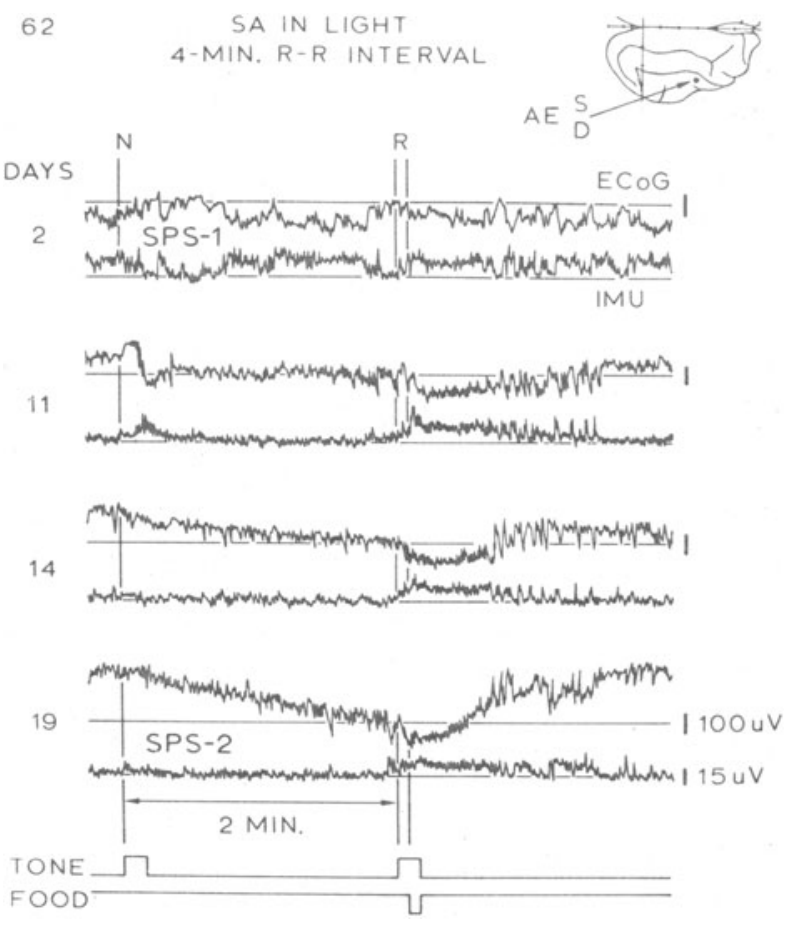

Fig. 2. Representative pairs of ECoG-IMU tracings for individual trials taken from days indicated illustrate the SPS-IMU relationships observed during SA training in S 62. Close SPS-IMU association (SPS-1) was characteristic during early acquisition (Day 2) and was obtained with $R$ and during post- $R$ intervals throughout training (right half of the figure). As the pre- $R$ ( $N$ to R) negative SPS was acquired (left half of the figure), it became increasingly dissociated from the IMU measure (SPS-2). Multiple unit integrator output is filtered from dc to half-amplitude frequency response at $1.5 \mathrm{~Hz}$ in all figures except $\mathrm{Fig}$. 8 . 
50-100 microV over the 2-min pre- $\mathrm{R}$ interval was not accompanied by a corresponding graded or sustained change in IMU level. By Day 19, each trial exemplified the fully developed pre-R SPS-2 relationship while preserving the SPS-1 relationship during and immediately after the $R$ trial. The reliability of this observation is demonstrated in Fig. 3 , in which each ECoG tracing (top group) shows a graded negative 2-min pre-R SPS of 200-300 microV. However, none of the corresponding IMU tracings (bottom) shows a corresponding change.

Not all SPS-IMU relationships observed may be unequivocally classified into SPS-1 vs SPS- 2 categories. For example, in the transition which occurred during acquisition training (Fig. 2) from association of SPS and IMU measures in the pre-R interval (SPS-1, Day 2) to dissociation of the two measures (SPS-2, Days 14 and 19), there are intermediate stages (Day 11) in which the measures show a partial dissociation, combining attributes of both SPS-1 and SPS-2 patterns. Another example is seen in the response to reinforcement (Figs. 1 and 2). On Day 2, a clear and distinct SPS-1 to food is not evident. It could be present but be obscured by continual SPS-1 related orienting responses to the novelty of the experimental situation, usually accompanied by bursts of licking activity. In later training (Days 11, 14, and 19), with the subsidence of such SPS-1 related orienting bheavior, a clear SPS to food, which is clearly associated with IMU activity, becomes evident. This shift, then, is like that of SPS-1 in having direct relation to stimuli and IMU activity, but is also like that of SPS-2 in being "acquired." Such patterns are presently interpreted as cases of mixed SPS-1 and SPS-2 dynamics rather than contradictions of the criteria differentiating the two. In addition, there are cases in which both patterns occur together but remain clearly distinguishable. In the $\mathrm{DR}_{3}$ and S-D tracings in Fig. 8, SPS-1 patterns are evident during saccade-related lambda-wave activity which is associated with IMU increments, but these transient SPS-1 events are superimposed on and readily distinguishable from the long-lasting pre-R SPS-2 slopes.

Figure 3 also shows the elicitation of a conditional IMU response (cortical CR) during the first $5 \mathrm{sec}$ of the CS when it signaled the US (i.e., during the CS-US interval at $\mathrm{R}$ ). However, a similar IMU CR occurred minimally or not at all during the first $5 \mathrm{sec}$ of the CS when it signaled no US (at N). Furthermore, this differential elicitation of IMU CRs by $\mathrm{N}$ vs $\mathrm{R}$ tones occurred in the absence of any significant differences in baseline IMU levels during 5-sec control intervals immediately prior to $\mathrm{N}$ vs $\mathrm{R}$ tones. These observations were statistically corroborated by application of one-tailed, related-measures $t$ tests contrasting pre- $\mathrm{N}$ vs pre-R IMU levels $(\mathrm{t}<1, \mathrm{df}=6)$ and IMU levels during the first $5 \mathrm{sec}$ of $\mathrm{N}$ vs $\mathrm{R}$ tones $(\mathrm{t}=2.74, \mathrm{df}=6, \mathrm{p}<.05)$. (The numbers on which these tests were based

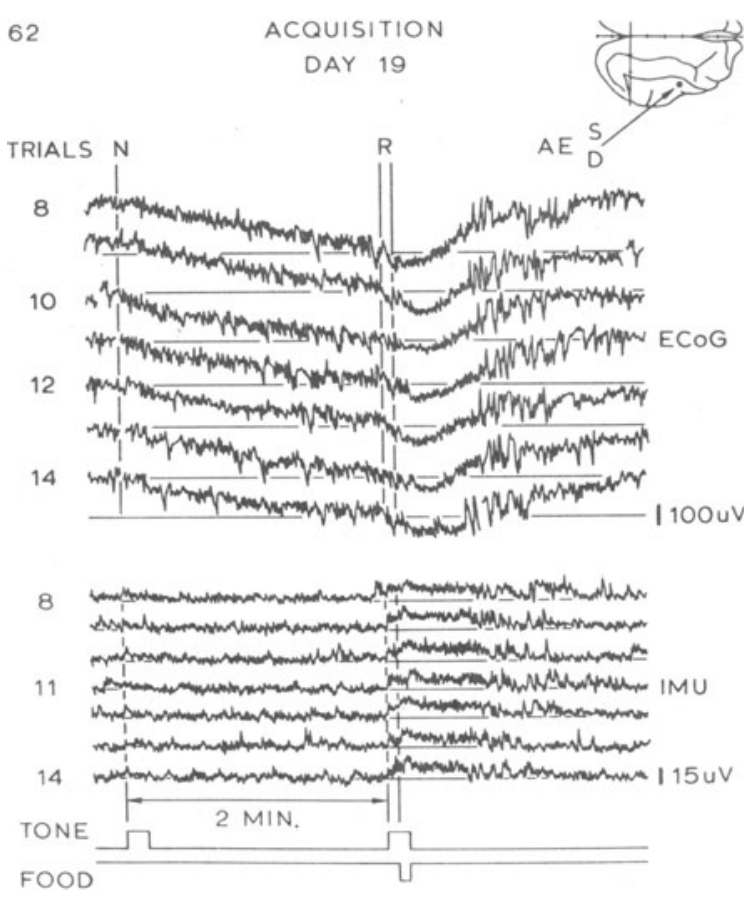

Fig. 3. Representative ECoG tracings of seven consecutive trials (28 min continuous recording) illustrate reliability of the SPS pattern (top) acquired under the SA schedule in S 62. In relation to corresponding IMU tracings (bottom), reliability of SPS-IMU dissociation (SPS-2) during the pre-R (N to R) interval and SPS-IMU association during the post-R ( $R$ to $N$ ) interval is demonstrated. IMU tracings also show SA of IMU CRs (see text).

represented area integrations under IMU curves computed from a common baseline extended throughout the 28-min recording period).

The four successful loci in S 62 (bilateral implants in $\mathrm{AE}$ and PM gyri) all showed acquisition of pre-R-negative/post-R-positive SPS patterns similar in their time course and dissociation from IMU to the relations illustrated from the right $\mathrm{AE}$ placement (Figs. 1, 2, and 3). Furthermore, all four loci showed clear evidence of single alternation of IMU CRs (as in Fig. 3) although the latter observation achieved statistical significance only for the two AE placements. Electrode deterioration (low conductivity, high dc offsets, and rapid drift) precluded recording from either of the bilateral pericruciate placements in this animal.

Of the four remaining Ss which received SA training, two showed acquisition of SPS patterning from one PM locus each (Fig. 5, top). Although clear dissociation of the IMU from the pre-R SPS (i.e., SPS-2) was also observed in these two animals, it was less reliable than that obtained from the AE loci of S 62 and occurred without statisically significant levels of SA of IMU CRs. Less complete disappearance of behaviorally related SPS-1 activity characterized the prereinforcement patterns of these animals.

Behaviorally, the licking response was maximal during 
and shortly following the food US (5 to 7 licks/sec), declining gradually to near-zero baseline levels within 1 to $3 \mathrm{~min}$ (mean $=1.5 \mathrm{~min}$ ) following the US. This observation is similar to the UR "after-discharge" functions reported for the rabbit's jaw-movement response (Sheafor \& Gormezano, 1970). However, the anticipatory lick response of the cat to the CS failed to show any reliable evidence of SA as has been demonstrated for the rabbit's jaw-movement response (Poulos et al, 1972). Although all five Ss showed high levels of anticipatory licking to both $\mathrm{N}$ and $\mathrm{R}$ tones during the first few sessions ( $80 \%$ to $90 \%$ ), anticipatory licking to both $\mathrm{N}$ and $\mathrm{R}$ tones declined to levels under $30 \%$ within 2 weeks of training and failed to show sustained, statistically significant levels of SA at any stage of training.

\section{Effect of Varying the R-to-R Interval}

After 55 days training under the original 4-min SA schedule, the effects of changing the basic R-R interval were studied in S 62. The interval was reduced to $2 \mathrm{~min}$ for 30 days and again to $1 \mathrm{~min}$ for 57 more days. Figure 4 shows that the animal acquired SPS patterns which conformed appropriately to the shorter intervals. Finally, the original 4 min R-to-R schedule was reintroduced for 35 days. The pattern characteristic of the previous 4-min SA training was reacquired. This occurred in all four loci of S 62. A sample of the short interval response acquired at a PM locus is shown in Fig. 6.

The relationships between SPS and licking activity observed over the whole course of these changes establish that the overall SPS pattern did not reflect patterning of licking behavior. At the 4-min R-to-R interval, the beginning of the pre-R negative SPS tended to coincide with cessation of licking activity. But at the 2-min R-to-R interval, licking continued well beyond the point of initiation of the pre-R negative SPS. At the 1-min R-to-R interval, licking activity was nearly incessant, continuing up to the moment of R-tone onset. At this point, it ceased abruptly during the 5-sec CS-US interval, beginning again at US onset. At these shorter R-to- $R$ intervals, the pre-CS IMU baseline was constantly elevated above that observed at the 4-min R-to-R interval (Fig. 3) and showed consistently greater variability at all loci. Thus, although clear evidence of SPS-IMU dissociation was obtained from the AE sites during pre- $\mathrm{R}$ negative shifting at the shorter intervals, it was much less dramatic than that demonstrated at the longer interval (Fig. 3). Similarly, although both AE loci continued to show SA of IMU CRs at the shorter intervals in terms of mean differences, in the context of greater IMU variability these differences yielded statistically nonsignificant outcomes. In the PM loci, at the 1-min interval, the more continuous elevation of the IMU baseline between reinforcements enabled the

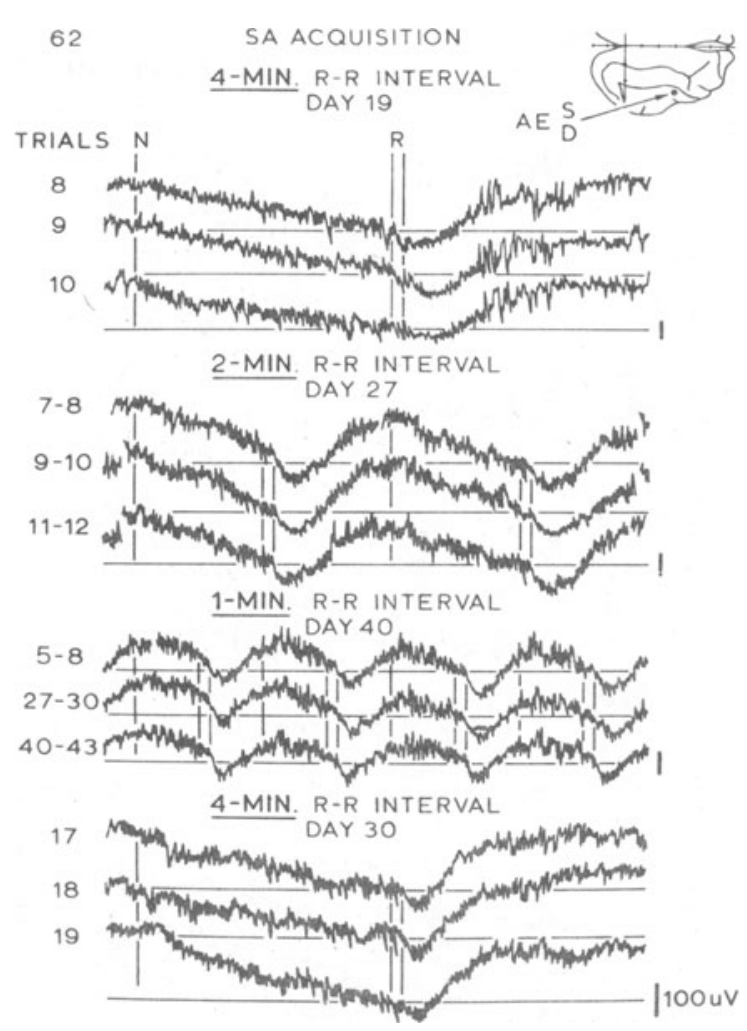

Fig. 4. Representative ECoG tracings of consecutive trials during 55 days SA training at the original 4-min R-to-R (R-R) interval, following reduction to $2 \mathrm{~min}$ for $\mathbf{3 0}$ days, to $1 \mathrm{~min}$ for 57 days, and finally, following reinstatement of the original 4-min R-R interval for 35 days (top to bottom).

observation of a reduction of IMU activity at the time of food delivery and shortly thereafter. This was inverse to the IMU increment seen with food delivery in the 4-min SA training. In the 4-min SA training, food delivery occurred in the context of low holding IMU level and no licking activity.

The R-R interval controls the period of the acquired SPS pattern for intervals up to $9 \mathrm{~min}$ as observed in the sixth S (172) which was offered food reinforcements in a small cup. When offered food reward every $9 \min$ (not on SA schedule), this $\mathrm{S}$ showed gradual acquisition of an anticipatory pre- $R$ negative SPS in two of three PM intracortical sites recorded against a common reference in ventricle. When the R-R interval was increased from 9 to $30 \mathrm{~min}$, the previously observed anticipatory shift was gradually extinguished over 6 daily sessions. When the 9-min R-R interval was reinstated, the former pre-R negative shift was rapidly reacquired over the next 3 days. No attempt was made to train the animal to a 30-min R-R interval. The anticipatory negative SPSs acquired at the 9-min R-R interval were not accompanied by corresponding changes in IMU level, exemplifying the SPS-2 type of relationship seen in three other Ss. The parallel recording of the third PM 


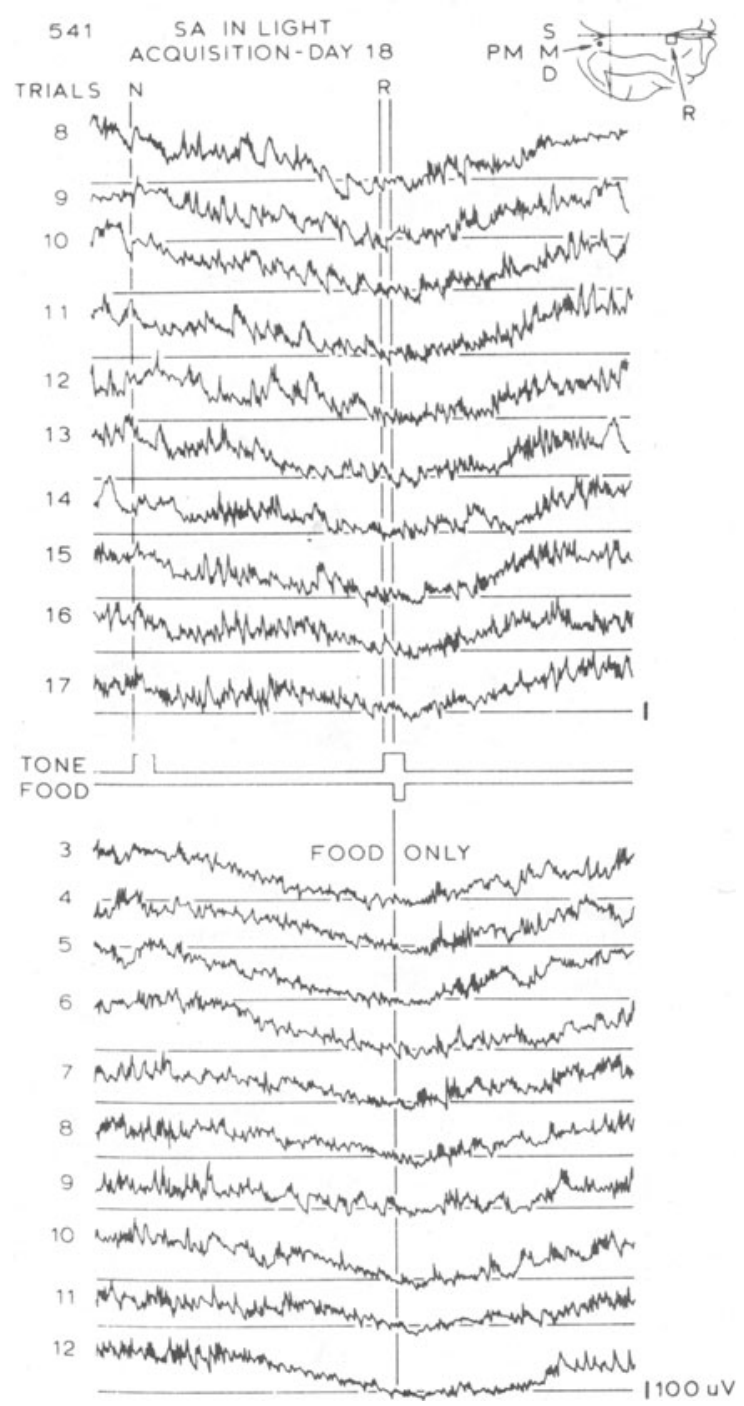

Fig 5. Representative ECoG tracings of 10 consecutive trials (40 min continuous recording) derived $D$ to skull reference at $R$ illustrate reliability of the SPS pattern acquired during SA training (top) in S 541. Tone onset-to-onset $=2 \mathrm{~min}$. Bottom tracings demonstrate that deletion of the CS produced little or no disruption of the 4-min SPS pattern. (Open squares in this and subsequent figures indicate reference electrodes in or on skull bone).

placement showed no acquired SPS and supports exclusion of the ventricle reference as the source of shift in this animal.

\section{Effects of Omission of CS or US}

The top half of Fig. 5 demonstrates the reliability of the SPS pattern acquired by another S (541) following 18 days of training under the SA schedule. When all CSs were omitted and food US deliveries were maintained every $4 \mathrm{~min}$, the SPS pattern was minimally disrupted as demonstrated by the tracings in the bottom half of Fig. 5. The same manipulation yielded similar results when carried out at the 1-min R-R interval in S 62, as shown in Fig. 6. In both cases, the SPS pattern acquired during SA training was maintained independently of the CS schedule.

However, dependence of the acquired SPS pattern on regular occurrence of the food US was observed repeatedly as illustrated in Fig. 7. Omission of the food US beginning on Trial 14 immediately and completely obliterated the SPS pattern even though the tone-CS schedule was maintained throughout. When the food US was reintroduced at Trial 21, the SPS pattern was immediately restored.

The temporally conditioned cortical SPS pattern appears to reflect activity occurring mainly at the lower layers of the cortex. In Fig. 6, for example, cortical depth (D) recorded against a remote skull reference (D-R ${ }_{3}$ ) demonstrates the pre-R-negative/post-R-positive SPS pattern. Simultaneous recordings of the surface electrode with respect to the same reference $\left(S-R_{3}\right)$ and an interreference derivation $\left(\mathbf{R}_{\mathbf{4}}-\mathbf{R}_{\mathbf{3}}\right)$ showed a virtual

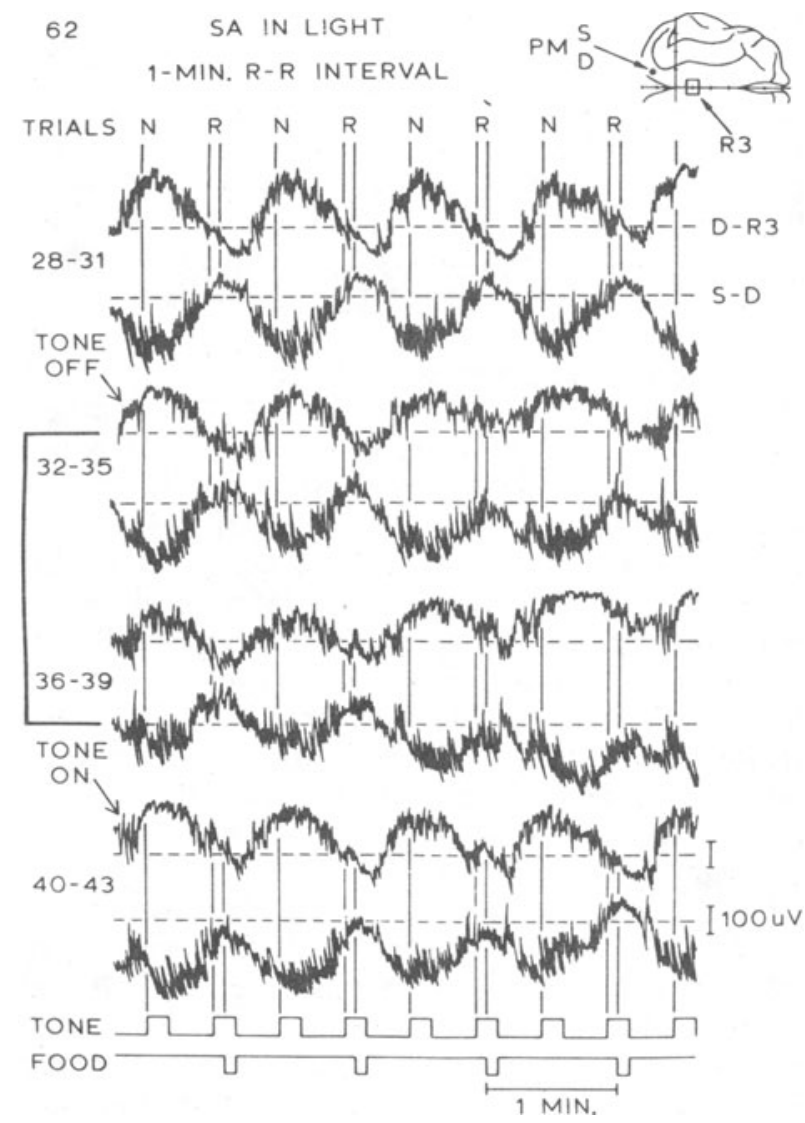

Fig. 6. Representative ECoG tracings of 16 consecutive trials from S 62 illustrate that deletion of the CS (Trials 32-40) produced minimal disruption of the SPS pattern acquired at the 1-min R-R interval. Potential differences between $D$ and $S$ differ relatively little from those recorded between $D$ and $R_{3}$, the phase inversion being only the consequence of polarity inversion at the amplifier inputs. This, with relations shown in Fig. 8, indicates dominant influence in pre-R SPS is negativity at D. 
absence of SPS activity (Fig. 8). Similar results were obtained for two other loci in S 62. Its fourth placement (right AE) varied in showing maximal activity in the surface lead. Acquired SPS patterns shown by the other Ss were similarly interpretable as reflecting activity at the lower cortical layers. In visual placements, the pre-R depth negative SPS was of the same polarity as that of the saccade-related lambda wave, as illustrated in Fig. 8.

Localization problems have yet to be resolved. Although arrays are aimed for the simplest orientation of tips along an axis normal to the pial surface and parallel to cortical columns, histology revealed that tracks often passed $1 / 2$ to $1 \mathrm{~mm}$ into subjacent white matter or arrived at various levels of cortical columns not parallel to the track. Further fractionation studies are in progress for the rigorous assignment of anatomical site and polarity to components of the acquired patterns described.

\section{DISCUSSION}

These observations confirm some SPS being clearly correlated with mass action potential activity. Such SPSs, which predominate in the untrained animal and occur during postreinforcement intervals throughout training, are similar to transient ECoG and short-duration (1-5 sec) SPS phenomena previously reported (Rebert, 1969, 1973a; Rowland \& Anderson, 1971; Rowland \& Dines, 1973). This kind of SPS, which

$$
\begin{aligned}
& 62 \\
& \begin{array}{c}
\text { SA IN LIGHT } \\
\text { 1-MIN. R-R INTERVAL }
\end{array}
\end{aligned}
$$

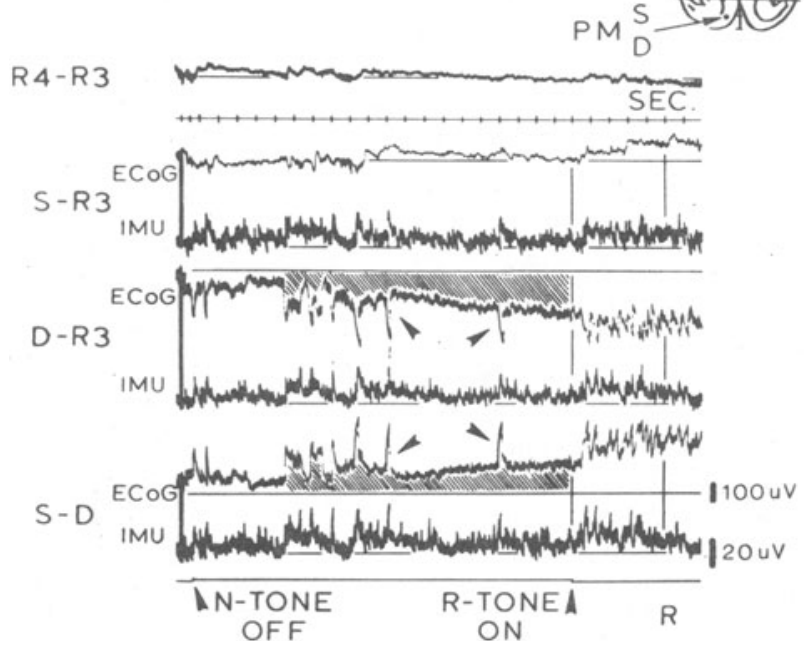

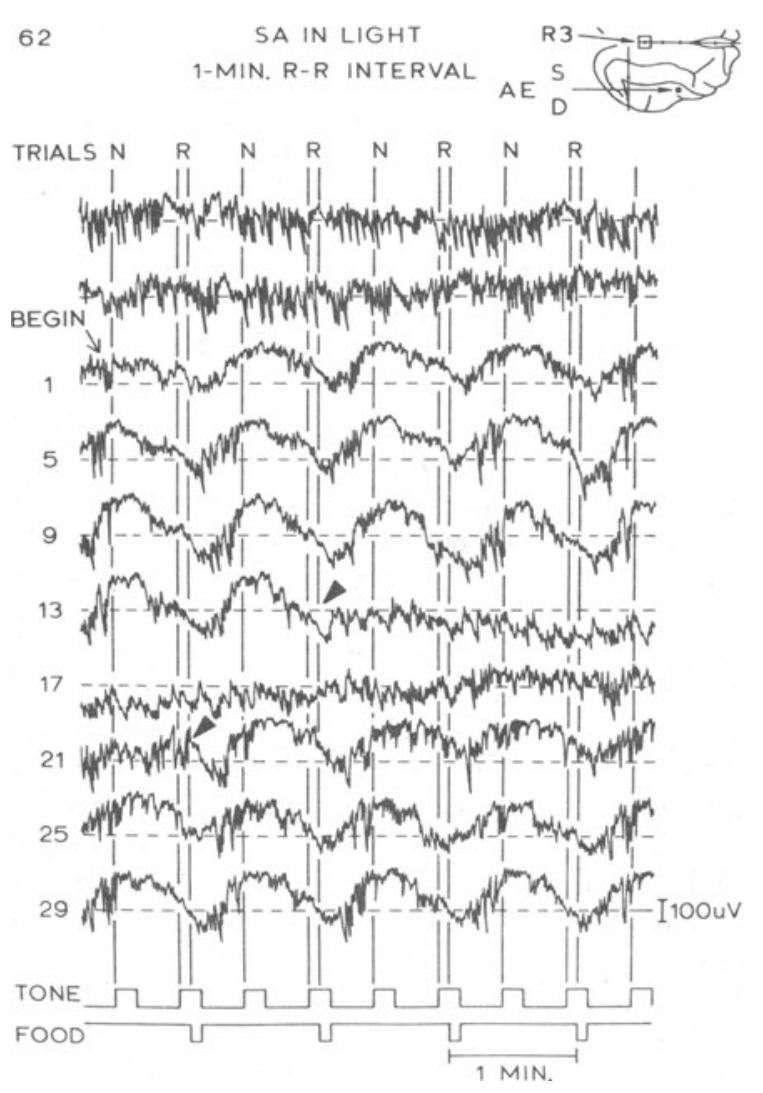

Fig. 7. ECoG recording (D-R 3 derivation) during an entire session illustrates immediate obliteration of the acquired SPS pattern by omission of the food US (Trials 14-20) and its immediate restoration by reintroduction at Trial 21 (see arrows) of the food US. The tone CS schedule continued throughout. Also note absence of pattern in tracings of top two lines before SA schedule begins.

is not acquired and is clearly correlated with mass action potential activity, is identified as SPS-1.

The unexpected observation is that the acquired large graded SPSs which occurred systematically during prereinforcement intervals were unattended by corresponding change in mass action potential activity. This kind of SPS, occurring in the absence of direct evoking stimuli, or any change in mass action potential activity, is identified as SPS-2. This dissociation of SPS from action potential activity requires ruling out possible artifactual explanations. Preserved dynamic sensitivity of the mass action potential record is, of course, essential to the claim that the observed nonreactivity of the units is significant. Dynamic

Fig. 8. Simultaneous recordings from S 62 during SA training at the 1-min R-R interval. Note expanded time base. ECoG and MU integrator outputs filtered from dc to half-amplitude frequency response at $40 \mathrm{~Hz}$. D-R 3 and S-D ECoG recordings (shaded) are the same derivations as those displayed in Fig. 6 at a slower paper speed and greater filtering. Shaded D-R 3 and S-D ECog recordings illustrate pre-R cortical depth negative SPSs in the same polarity as saccade-related lambda waves (indicated by arrows). Note relative absence of both SPS and lambdas in S-R 3 ECoG and interreference $\left(R_{4}-R_{3}\right)$ recordings (equalized gains) and ECoG-IMU correlation during lambda waves in $D-R_{3}$ and $S-D$ recordings. Although saccade-related IMU increments are seen at the surface ( $\left.S-R_{3}\right)$, the lambda wave itself is relatively absent in the surface ECoG recording. 
sensitivity of action potential recording is supported by the observations that: (1) throughout the acquisition period in which the prereinforcement SPS-2 developed, IMU response continued to appear at the onset of the $\mathbf{R}$ tone and throughout the postreinforcement interval; (2) the units record remained highly responsive to novel arousal-inducing stimuli throughout the acquisition period; and (3) the low background average level of unit activity continued throughout to show clear reduction with spontaneous drowsiness and ECoG synchrony.

Several bases for failing to detect changes in mass action potential activity may be entertained. First, cortical action potential generators closely related to the observed SPS may be too remote from the electrode to appear in the IMU record. To date, however, no cortical locus has shown IMU activity correlated with the protracted and graded pre-R SPS. A second possibility, that action potential generators in subcortical regions are generating the SPS-2, requires the assumption that the IMU record is insensitive to a particular subpopulation of corticipetal action potentials. The sensitivity of the IMU record in the SPS-1 situation suggests the possibility that the IMU is responsive to specific system activation, whereas it may remain unresponsive to a more diffuse system influence. This would be consistent with Jasper's (1960) observations that stimulation of unspecific thalamic areas may give rise to widespread cortical recruiting responses but without unit firing detectable at any depth in the cortex with microelectrodes. Diffuse system influence is consistent with the pattern of SPS-2 response being quite similar in both auditory and visual cortex (cf. Figs. 4 and 6). Thus, a subpopulation of action potential generators, either cortical or subcortical, which are peculiarly capable of producing the SPS-2 may be too small in magnitude, or too dispersed temporally or spatially to be detected in the mass unit recording. A third, less parsimonious, possibility is that a subpopulation of action potential generators becomes active while an equal subpopulation reciprocally becomes inactive producing the SPS-2 without a net change in the IMU profile of the whole population. A fourth possibility is exclusion by the high-frequency bandpass (.8 to $2.6 \mathrm{kHz}$ ) of fast-rising short-duration (ca 100 microsec) action potentials, termed "Golgi-cell spikes" by Mountcastle et al (1969), which might be important in SPS-2 genesis. Preliminary observations indicate that extending the bandpass to $60 \mathrm{kHz}$ increases low-amplitude electronic noise, elevating the IMU baseline and slightly obscuring the "dynamic profile" of the IMU record because of the reduced signal to noise ratio. No enhancement of IMU "profile" changes attributable to admitting higher frequencies was observed.

If future studies fail to reveal cortical action potential changes correlated with pre-R SPS-2, at least two bases for such dissociation may be considered: (1) a neuronal origin of SPS-2 involving dendrosomatic activity relatively autonomous with respect to action potential activity, or possibly involving a subpopulation of "silent" (nonspiking) interneurons; and (2) a nonneuronal cell population, for example, presumed astrocytic glia.

Against dendrosomatic activity in spiking neurons is the observation that SPS-1 amplitudes smaller than those seen in SPS-2 are tightly coupled with marked IMU changes. However, rate of change of potential may be a more important factor in action potential genesis than the amplitude reached, and the possibility of spread of electrotonic potential among the dendrodendritic arborizations of neurons is encouraged by the demonstration of dendrodendritic tight junctions (Sloper, 1971, 1972). There is also the possibility that the SPS-2 may reflect the graded activity of unmyelinated short-axoned, or anaxonic microinterneurons. That such interneurons may constitute the associative and memory-storage elements of the brain has been repeatedly suggested (cf. Altman, 1967; Horridge, 1968), and the acquired character of the SPS-2 would be consistent with this notion.

The leading candidate for a nonneuronal cellular source is the presumed astrocytic glia. Ransom and Goldring $(1973 a, b, c)$ have extended earlier observations showing dependence of cortical SPSs on protracted slow depolarizations and subsequent hyperpolarizations of the glial membrane following brief bursts of neuronal firing. The slow glial membrane responses, however, have been shown to depend upon increases and subsequent decreases in extracellular potassium levels following depolarization and subsequent hyperpolarization of neuronal membranes. While such a mechanism might account for the SPS-1 relationships observed in the untrained animal and during postreinforcement intervals, it could not account for the protracted SPS-2 observed during the 2-min prereinforcement interval unless it could be demonstrated either that: (1) extracellular potassium levels were slowly increasing during the prereinforcement interval, due perhaps to graded depolarization of neuronal membranes, but without significant action potential genesis; or (2) the glial membranes are progressively depolarized by some other mechanism. The first alternative is open to test by use of a potassium electrode recording simultaneously with the SPS-2 changes. Test of the second alternative comes to depend on intraglial recording during the pre-R SPS-2.

Whatever the mechanisms of SPS genesis, the present observations suggest that cortical SPS provides distinctive information about brain mechanisms in learning and performance which do not appear in concurrent recordings of mass action potential activity. The present demonstration of single alternation of IMU CRs, although interesting, may well be as "peripheral" a reflection of the learning going on in this situation as the demonstrations by Poulos et al of single alternation of 
behavioral CRs (cf. Miller, 1961). In both cases, differential elicitation of CRs by the same CS is observed depending upon whether the CS occurs in a context signaling nonreinforcement (at $\mathrm{N}$ ) or a context signaling reinforcement (at $\mathrm{R}$ ), but the level of response to a particular CS cannot be predicted from the baseline activity level prior to the CS. As outlined in the introduction, many theorists have appealed to contextually conditioned "expectancies" of nonreinforcement (at N) and reinforcement (at R) which serve alternately to inhibit response to the CS in the $\mathrm{N}$ context and to facilitate responding to the CS in the R context (e.g., Amsel, 1967; Bolles, 1972; Rescorla \& Wagner, 1972). A similar notion is expressed by Tanji and Evarts (1973) who report that the reflex response of a cortical neuron can be "gated" on or off depending upon the acquired "set" or "expectancy" of the monkey. The fact that the level of the acquired cortical SPS pattern relates systematically to the contexts of nonreinforcement and reinforcement prior to CS presentations strongly suggests that this measure may reflect contextually conditioned expectancies which are not reflected either in IMU recording or in overt behavior (Poulos et al, 1972) but which may significantly determine the level of IMU and behavioral responses to the CS. ${ }^{1}$

\section{REFERENCES}

Altman, J. Postnatal growth and differentiation of the mammalian brain with implications for a morphological theory of memory. In G. C. Quarton, T. Melnechuk, and F. O. Schmitt (Eds.), The neurosciences: A study program. New York: Rockefeller University Press, 1967. Pp. 723-743.

Amsel, A. Partial reinforcement effects on vigor and persistence. In K. W. Spence and J. T. Spence (Eds.), The psychology of learning and motivation: Advances in research and theory. Vol. 1. New York: Academic Press, 1967. Pp. 1-65.

Bolles, R. C. Reinforcement, expectancy, and learning. Psychological R eview, 1972, 79, 394-409.

Capaldi, E. J. A sequential hypothesis of instrumental learning. In K. W. Spence and J. T. Spence (Eds.), The psychology of learning and motivation: Advances in research and theory. Vol. 1. New York: Academic Press, 1967. Pp. 67-156.

Chiorini, J. R. Slow potential changes from cat cortex and classical aversive conditioning. Electroencephalography \& Clinical Neurophysiology, 1969, 26, 399-406.

Donchin, E., Otto, D., Gerbrandt, L. K., \& Pribram, K. H. While a monkey waits: Electrocortical events recorded during the foreperiod of a reaction time study. Electroencephalography \& Clinical Neurophysiology, 1971, 31, 115-127.

Galambos, R. A glia-neural theory of brain function. Proceedings of the National Academy of Science, U.S., 1961, 47, 129-136.

Grossman, R. G., Whiteside, L., \& Hampton, T. L. The time course of evoked depolarization of cortical glial cells. Brain Research, 1969, 14, 401-415.

Horridge, G. A. Interneurons, London: Freeman, 1968.

Irwin, D. A., and Rebert, C. S. Slow potential changes in cat brain during classical appetitive conditioning of jaw movements using two levels of reward. Electroencephalography \& Clinical Neurophysiology, 1970 , 28, 119-126.

Jasper, H. H. Unspecific thalam ocortical relations. In J. Field, H. W. Magoun, and V. E. Hall (Eds.), Handbook of Physiology, Section 1. Neurophysiology, Vol. 1. Baltimore, Waverly Press 1960.

Karahashi, Y., \& Goldring, S. Intracellular potentials from "idle" cells in cerebral cortex of cat. Electroencephalography \& Clinical N europhysiology, 1966, 20, 600-607.

Kuffler, S. W., \& Nicholls, J. G. The physiology of glial cells. Ergebnisse der Physiologie, Biologischen Chemie \& Experimentellen Pharmakologie, 1966, 57, 1-90 (In English). Marczynski, T. J. Lysergic acid diethylamide (LSD) mimics the effect of diffuse light input on EEG correlates of conditioned operant behavior. Experimental Neurology, 1972, 34, 255-263.

Miller, N. E. Integration of neurophysiological and behavioral research. Annals of the New York Academy of Sciences, $1961,830-839$.

Mountcastle, V. G., Talbot, W. H., Sakata, H., \& Hyvarien, J. Cortical neuronal mechanisms in flutter-vibration studied in unanesthetized monkeys: Neuronal periodicity and frequency discrimination. Journal of Neurophysiology, 1969, 32, 452-484.

Pavlov, I. P. Conditioned reflexes. (Translated by G. V. Anrep.) London: Oxford University Press, 1927.

Poulos, C. X., Sheafor, P. J., and Gormezano, I. Classical appetitive conditioning of the rabbit's (ory ctolagus cuniculus) jaw-movement response with a single alternation schedule. Journal of Comparative \& Physiological Psychology, 1971, 75, 231-238.

Ransom, B. R., \& Goldring, S. Ionic determinants of membrane potential of cells presumed to be glia in cerebral cortex of cat. Journal of Neurophysiology, $1973 \mathrm{a}, 36,855-868$.

Ransom, B. R., \& Goldring, S. Slow depolarization in cells presumed to be glia in cerebral cortex of cat. Journal of Neurophysiology, $1973 \mathrm{~b}, 36,869-878$.

Ransom, B. R., \& Goldring, S. Slow hyperpolarization in cells presumed to be glia in cerebral cortex of cat. Journal of Neurophysiology, 1973c, 36, 879-892.

Rebert, C. S. DC and multiple-unit recording in lateral geniculate body of the cat. Proceedings of the $77 \mathrm{th}$ Annual American Psychological Association Convention, 1969, 77, 215-216.

Rebert, C. S. Cortical and subcortical slow potentials in the monkey's brain during a preparatory interval Electroencephalography \& Clinical Neurophysiology, 1972, 33, 389-402.

Rebert, C. S. A technique for simultaneous measurement of DC and multiple unit responses. Electroencephalography \& Clinical Neurophysiology, 1973a, 34, 326-328.

Rebert, C. S. Slow potential correlates of neuronal population responses in the cat's lateral geniculate nucleus. Electroencephalography \& Clinical Neurophysiology, 1973b, 35, 511-515.

Rebert, C. S., \& Irwin, D. A. Simple electrode configurations for chronic or acute recording of DC potentials from subcortical nuclei of the brain. Electroencephalography \& Clinical Neurophysiology, 1973, 34, 440-442.

Rebert, C. S., \& Irwin, D. A. Slow potential changes in cat brain during classical appetitive and aversive conditioning of jaw movement. Electroencephalography \& Clinical Neurophysiology, 1969, 27, 152-161.

Rescorla, R. A., \& Wagner, A. R. A theory of Pavlovian conditioning: Variations in the effectiveness of reinforcement and nonreinforcement. In A. H. Black and W. F. Prokasy (Eds.), Classical conditioning II: Current theory and research. New York: Appleton-Century-Crofts, 1972. Pp. 64-99.

Rowland, V. Steady potential phenomena of cortex. In G. C. Quarton, T. Melnechuk, and F. O. Schmitt (Eds.), The neurosciences: A study program. New York: Rockefeller University Press, 1967. Pp. 723-743.

Rowland, V. Cortical steady potential (direct current potential) in reinforcement and learning. In E. Stellar and J. M. Sprague (Eds.), Progress in physiological psychology. Vol. 2. New York: Academic Press, 1968. Pp. 1-77.

Rowland, V. Cortical steady potential shifts and mass unit activity in relation to expectancy (Abstract). Conditional R eflex, 1973, 8, 167-168.

Rowland, V., In A. Katchalsky, V. Rowland, and R. Blumenthal (Eds.) Dynamic patterns of brain cell assemblies. Cambridge: MIT Press, 1974. Pp. 111-121.

Rowland, V., \& Anderson, R. Brain steady potential shifts. In E. Stellar and J. M. Sprague (Eds.), Progress in physiological psychology. Vol. 4. New York: Academic Press, 1971. Pp. 37-51.

Rowland, V., \& Dines, G. Cortical steady potential shift in relation to the rhythmic electrocorticogram and multiple unit activity. In R. F. Thompson and M. M. Patterson (Eds.), Bioelectric recording techniques: Part $A$, cellular processes and brain potentials. New York: Academic Press, 1973. Pp. 369-385.

Sheafor, P. J., \& Gormezano, I. Conditioning the rabbit's (oryctolagus cuniculus) jaw-movement response: US magnitude effects on URs, CRs, and pseudo-CRs. Journal of Comparative \& Physiological Psychology, 1972, 81, 449-456.

Sloper, J. J. Dendro-dendritic synapses in the primate motor cortex. Brain $R$ esearch, 1971, 34, 186-192.

Sloper, J. J. Gap junctions between dendrites in the primate neocortex. Brain R esearch, 1972, 44, 641-646.

Somjen, G. G. Evoked sustained focal potentials and membrane potential of neurons and unresponsive cells of the spinal cord. Journal of Neurophysiology, 1970, 33, 562-582. 
Steinmetz, G. T., \& R ebert, C. S. Postreinforcement changes of steady potentials in premotor cortex of monkeys. Physiology \& Behavior, 1972, 9, 769-772.

Tanji, J., \& Evarts, E. V. Gating of motor cortex reflexes by prior instructions. Paper read at the Third Annual Meeting of the Society for Neuroscience, San Diego, California, November 1973.

\section{NOTE}

1. Authors express appreciation for the helpful review of
Henry Gluck, Marvin Wasman, and John Ferguson and for the technical assistance of Dennis Quinn, Kate Gruen, and Suzanne McKnight. Our thanks also to J. P. Rosenfeld and A. Rudell for instruction on implanting the transnasal cannula.
(Received for publication May 9, 1974; accepted May 23, 1974.) 